\title{
Behavior of a Multi-User MIMO System in Time-Varying Environments*
}

\author{
Yasutaka OGAWA $^{\dagger a}$, Fellow, Kanako YAMAGUCHI ${ }^{\dagger}$, Student Member, Huu Phu BUI ${ }^{\dagger \dagger}$, Nonmember, $^{\dagger}$ \\ Toshihiko NISHIMURA ${ }^{\dagger}$, and Takeo OHGANE ${ }^{\dagger}$, Members
}

\begin{abstract}
SUMMARY We evaluated the behavior of a multi-user multiple-input multiple-output (MIMO) system in time-varying channels using measured data. A base station for downlink or broadcast transmission requires downlink channel state information (CSI), which is outdated in time-varying environments and we encounter degraded performance due to interference. One of the countermeasures against time-variant environments is predicting channels with an autoregressive (AR) model-based method. We modified the AR prediction for a time division duplex system. We conducted measurement campaigns in indoor environments to verify the performance of the scheme of channel prediction in an actual environment and measured channel data. We obtained the bit-error rate (BER) using these data. The AR-model-based technique of prediction assuming the Jakes' model was found to reduce BER. Also, the optimum AR-model order was investigated by using the channel data we measured.

key words: multi-user MIMO system, channel state information, timevarying environment, channel prediction, autoregressive (AR) model-based prediction
\end{abstract}

\section{Introduction}

A tremendous amount of research has been done on multiple-input multiple-output (MIMO) systems over the last decade because they provide transmission at high data rates without increasing the frequency bandwidth [1]-[3]. MIMO systems achieve high-speed wireless transmission through space-domain signal processing. Adaptive antennas that also exploit space-domain signal processing had been studied for various applications before MIMO systems emerged [4]-[6]. MIMO systems are considered to be an extension of adaptive antennas but they have two clearly distinct aspects that are not seen in adaptive antennas.

First, high-speed transmission by MIMO systems is proved from the view-point of information theory [7]. Let us assume that we have a single channel between a transmitter and a receiver, and that the signal-to-noise power ratio at the output of the channel is $S / N$. The capacity of the channel is given by $C=\log _{2}(S / N+1) \simeq \log _{2} S / N$

Manuscript received February 26, 2013.

Manuscript revised May 20, 2013.

${ }^{\dagger}$ The authors are with the Graduate School of Information Science and Technology, Hokkaido University, Sapporo-shi, 0600814 Japan.

${ }^{\dagger \dagger}$ The author is with the National Key Lab of Digital Control \& System Engineering, University of Technology, Vietnam National University, Hochiminh City, Vietnam.

${ }^{*}$ The work of H.P. Bui was supported in part by the Vietnam National Foundation for Science and Technology Development (NAFOSTED) under Grant 102.02-2011.23.

a)E-mail: ogawa@ist.hokudai.ac.jp

DOI: 10.1587/transcom.E96.B.2364
bps/Hz when $S / N \gg 1$. If the transmit (TX) power is doubled, the signal-to-noise power ratio is also doubled, and the capacity is $\log _{2}(2 S / N+1) \simeq C+1 \mathrm{bps} / \mathrm{Hz}$. This means that although the TX power is doubled, the channel capacity is increased by only $1 \mathrm{bps} / \mathrm{Hz}$, which is not efficient. If we have two parallel equivalent channels, the capacity is $2 \log _{2}(S / N+1) \simeq 2 C$ bps/Hz. MIMO systems achieve parallel channels between a transmitter and a receiver in a space, and achieve high channel capacity.

Second, MIMO systems work properly in multipathrich environments because the correlations between channels are low, and the previously mentioned parallel transmission effectively improves communication. This means that MIMO systems exploit multipath propagation, which had been a nuisance to wireless communications because it caused fading. The authors believe that exploiting multipath propagation is a distinct paradigm shift.

It is true that MIMO systems are applications of antenna engineering. However, they are closely related with many other fields such as communications, signal processing, information theory, and radio propagation. The development of MIMO systems is a beneficial achievement from these techniques.

If a transmitter has channel state information (CSI), sophisticated transmission such as eigen-beam space division multiplexing (E-SDM) can be applied [8], which is also called a singular value decomposition (SVD) system [9] or MIMO eigenmode transmission system [10]. CSI is needed for downlink (DL) or broadcast transmission in multi-user MIMO systems [11]-[13]. CSI in a frequency division duplex (FDD) system must be fed back from mobile stations (MSs) to a base station (BS). CSI in time-varying environments may be outdated because of feedback delay. The effect of feedback delay is a critical issue, and analytical results have been reported in the literature [14]. Measurementbased considerations on the effect of outdated channels have been done by Wallace et al. [15]. We can obtain downlink CSI from the uplink signal in a time division duplex (TDD) system. We have the outdated CSI issue even in a TDD system when the time interval between the uplink channel and the downlink transmission cannot be neglected.

Multi-user MIMO systems are much more affected by outdated CSI than single-user ones. A BS needs to send signals using TX or beamforming weights that suppress interuser interference (IUI) and inter-stream interference (IStI) because MSs have fewer antennas than a BS and cannot sup- 
press this interference. The channels in time-varying environments at the actual time of transmission differ from those used to determine the TX weights. Thus, MSs suffer from interference, and transmission is seriously degraded.

A transmission scheme based on channel distribution information has been proposed to mitigate the effect of outdated CSI. Although communication is not optimum, it is stable in time-varying environments because the transmission scheme does not need CSI [16], [17]. Another countermeasure against time-variant environments is achieved by predicting channels. If we can predict future channels, the outdated CSI issue is resolved. Several schemes have been reported for channel prediction [18]-[25]. Of these, an autoregressive (AR) model-based method, which is often referred to as a linear predictor, is a typical scheme. Detailed considerations on the AR-model-based method are stated in the literature [18] and the references therein. We need to obtain AR coefficients to apply the method and knowledge about channel autocorrelations is required to compute these. One method of obtaining the autocorrelations is to calculate them from past channel information. If we have many channel data, we have accurate channel autocorrelations. However, if we do not have enough data, we may have poor autocorrelations. An other method is to calculate the channel autocorrelations assuming the Jakes' model. If we know the maximum Doppler frequency, the channel autocorrelations can easily be obtained. However, the Jakes' model is not always valid in actual radio environments.

We examined the prediction of channels with the ARmodel-based method for multi-user MIMO downlink transmission. We conducted measurement campaigns in the 5.2$\mathrm{GHz}$ frequency band in indoor environments and obtained a large number of statistically stationary time-varying channels [26]. We investigated the bit error rate (BER) by using the data.

The paper is organized as follows. The next section briefly describes the prediction of channels with the ARmodel-based method. Section 3 then presents the measuring setup for our experiment. Section 4 shows autocorrelations and Doppler spectra of the measured channels, and Sect. 5 explains our evaluation of BER. Finally, Sect. 6 prsents the conclusions we drew.

\section{AR-Model-Based Prediction of Channels}

We represent the channel from the $j$ th TX antenna of the BS to the $i$ th receive $(\mathrm{RX})$ antenna of the $k$ th MS at time $t$ by $h_{i j, k}(t)$. Future channel $h_{i j, k}\left((n+1) T_{f}\right)$ is predicted in ARmodel-based algorithms using $p$ previous channel values $h_{i j, k}\left(n T_{f}\right), h_{i j, k}\left((n-1) T_{f}\right), \ldots, h_{i j, k}\left((n+1-p) T_{f}\right)$, which are given by

$$
\hat{h}_{i j, k}\left((n+1) T_{f}\right)=\sum_{m=1}^{p} a_{m} h_{i j, k}\left((n+1-m) T_{f}\right) .
$$

Here, $a_{m}$ are AR coefficients, and $p$ is called the AR-model order [18], [27]. Although the AR coefficients are time- variant values, we assume channels are slowly varying and we can consider that they are independent of time for a certain period. The optimum AR coefficients are given by the Yule-Walker equation

$$
\boldsymbol{R} a=r .
$$

$\boldsymbol{a}$ is the $p$-dimensional vector in the above equation defined as

$$
\boldsymbol{a}=\left[\begin{array}{llll}
a_{1} & a_{2} & \cdots & a_{p}
\end{array}\right]^{T},
$$

where [ $]^{T}$ denotes the transpose. $\boldsymbol{R}$ is the $p \times p$ correlation matrix whose $l_{1}$ th row and $l_{2}$ th column element is given by

$$
\begin{aligned}
& r\left(l_{1}-l_{2}\right)=r^{*}\left(l_{2}-l_{1}\right) \\
& =E\left\{h_{i j, k}^{*}\left(\left(n+1-l_{1}\right) T_{f}\right) h_{i j, k}\left(\left(n+1-l_{2}\right) T_{f}\right)\right\} \\
& =E\left\{h_{i j, k}^{*}\left(\left(n+1-\left(l_{1}-l_{2}\right)\right) T_{f}\right) h_{i j, k}\left((n+1) T_{f}\right)\right\},
\end{aligned}
$$

where ${ }^{*}$ denotes the complex conjugate and $E\{\}$ denotes the ensemble average. Also, correlation vector $r$ is defined as

$$
\boldsymbol{r}=\left[\begin{array}{llll}
r(1) & r(2) & \cdots & r(p)
\end{array}\right]^{T} .
$$

The $l$ th element is given by

$$
r(l)=E\left\{h_{i j, k}^{*}\left((n+1-l) T_{f}\right) h_{i j, k}\left((n+1) T_{f}\right)\right\} .
$$

We have assumed a TDD system such as HIPERLAN/2 in the remainder of this paper [28]. TDD systems are also standardized in addition to FDD ones in 3GPP LTE and mobile WiMAX [29], [30]. As seen in Fig. 1, uplink and downlink signals are transmitted with a period of $T_{f}$, which is the frame duration in the TDD system. The BS estimates the channels for the MSs using uplink ACK packets, and sends DL packets using the multi-user MIMO E-SDM scheme [26]. The above mentioned inter-user interference (IUI) is suppressed by block diagonalization [31]-[33]. We assumed that the ACK and DL packets were so short that we could neglect channel changes in the duration of packets.

Using the previous $p$ channels at $n T_{f},(n-1) T_{f}, \ldots,(n+$ $1-p) T_{f}$ estimated by the ACK packets, we predict the DL channel at $n T_{f}+\tau$, which is given by

$$
\hat{h}_{i j, k}\left(n T_{f}+\tau\right)=\sum_{m=1}^{p} a_{m}^{\prime} h_{i j, k}\left((n+1-m) T_{f}\right) .
$$

In this case, AR coefficients $a_{m}^{\prime}$ are obtained by using a slightly modified Yule-Walker equation

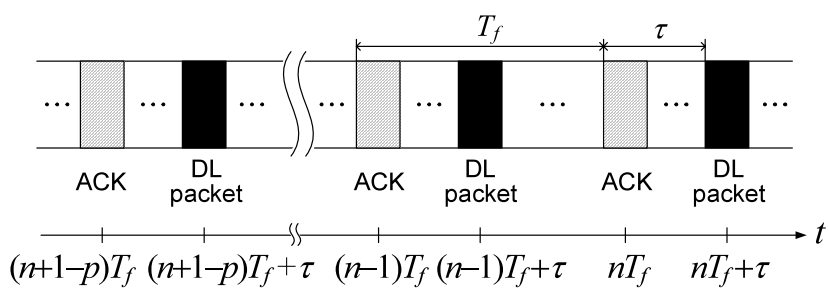

Fig. 1 TDD transmission frame format. 


$$
\boldsymbol{R} \boldsymbol{a}^{\prime}=\boldsymbol{r}^{\prime}
$$

Here, the $p$-dimensional vectors, $\boldsymbol{a}^{\prime}$ and $\boldsymbol{r}^{\prime}$, are defined as

$$
\begin{aligned}
\boldsymbol{a}^{\prime} & =\left[\begin{array}{llll}
a_{1}^{\prime} & a_{2}^{\prime} & \cdots & a_{p}^{\prime}
\end{array}\right]^{T} \\
\boldsymbol{r}^{\prime} & =\left[\begin{array}{llll}
r^{\prime}(1) & r^{\prime}(2) & \cdots & r^{\prime}(p)
\end{array}\right]^{T} .
\end{aligned}
$$

The $l$ th element of $\boldsymbol{r}^{\prime}$ is given by

$$
r^{\prime}(l)=E\left\{h_{i j, k}^{*}\left((n+1-l) T_{f}\right) h_{i j, k}\left(n T_{f}+\tau\right)\right\} .
$$

The channel correlation in the Jakes' model, without a constant coefficient, is given by

$$
E\left\{h_{i j, k}^{*}(t-T) h_{i j, k}(t)\right\}=J_{0}\left(2 \pi f_{\mathrm{D}} T\right),
$$

where $J_{0}(\cdot)$ is the zeroth-order Bessel function of the first kind and $f_{\mathrm{D}}$ is the maximum Doppler frequency. Thus, the $l_{1}$ th row and $l_{2}$ th column element of correlation matrix $\boldsymbol{R}$ is written as

$$
\begin{aligned}
r\left(l_{1}-l_{2}\right) & =r\left(l_{2}-l_{1}\right) \\
& =J_{0}\left(2 \pi f_{D}\left(l_{1}-l_{2}\right) T_{f}\right) .
\end{aligned}
$$

Also, the $l$ th element of correlation vector $\boldsymbol{r}^{\prime}$ is given by

$$
r^{\prime}(l)=J_{0}\left(2 \pi f_{\mathrm{D}}\left(\tau+(l-1) T_{f}\right)\right) .
$$

CSI in TDD systems is obtained by uplink ACK packets at the BS. However, downlink channel $h_{i j, k}\left(k T_{f}+\tau\right)$ required for correlation $r^{\prime}(l)$ given by (11) cannot be directly observed at the BS. The channel information must be fed back from the MS to the BS. This is a serious problem. However, if the channels can be approximated with the Jakes' model, we can calculate correlation matrix $\boldsymbol{R}$ and vector $\boldsymbol{r}^{\prime}$ using maximum Doppler frequency $f_{\mathrm{D}}$. We will demonstrate BER performance with the AR-based prediction of channels assuming the Jakes' model later.

\section{Measurements of Time-Varying Channels}

The measurement campaign for the multi-user MIMO system was carried out in a meeting room in a building of the Graduate School of Information Science and Technology at Hokkaido University, as shown in Fig. 2. The measurement was the same as that in Bui et al. [26]. We will repeat almost the same description here. The walls of the room were mostly plaster-board. The room also had reinforced concrete pillars, metal doors, and a metal whiteboard, where a 4-element TX and two 2-element RX linear arrays were placed on three tables. The TX and RXs corresponded to the BS and MSs stated in the previous sections. The arrays consisted of omnidirectional collinear antennas. The nominal gain of these antennas on the horizontal plane was about $4 \mathrm{dBi}$. The distances from the TX to RX1 and RX2 were $4 \mathrm{~m}$, while the spacing between RX1 and RX2 was $3 \mathrm{~m}$. The channels were measured for all the TX and the RX antenna pairs with a vector network analyzer. The measurement band was from 5.15 to $5.40 \mathrm{GHz}$ (bandwidth =

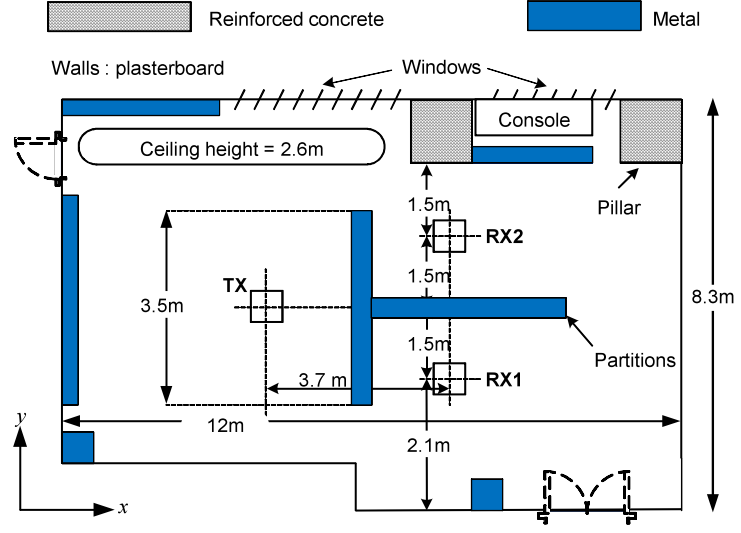

Fig. 2 Measurement site (top view). (Reprinted from Bui et al. [26] (C)2013 IEEE)
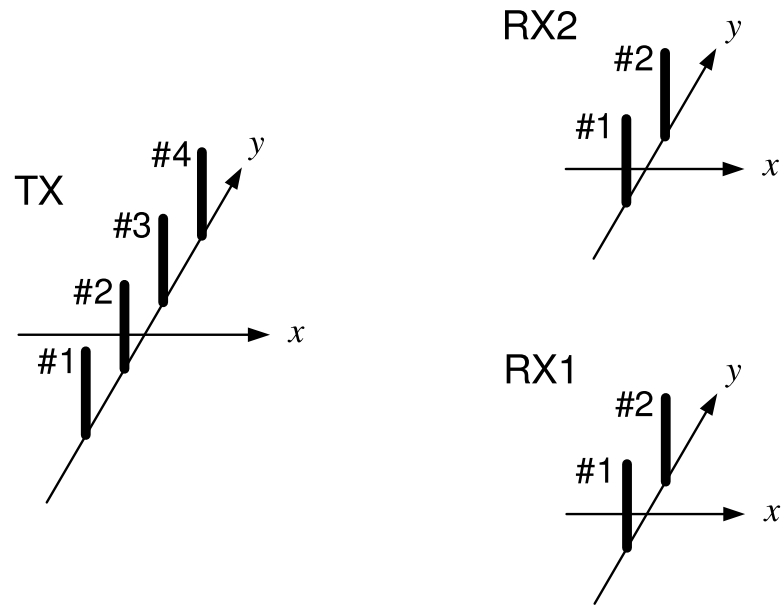

Fig. 3 Array orientations

$250 \mathrm{MHz}$ ), and we obtained 1,601 frequency domain data with $156.25 \mathrm{kHz}$ intervals. The antenna spacing was $3 \mathrm{~cm}$ (half-wavelength at $5 \mathrm{GHz}$ ).

The array orientation was along the $y$-axis as shown in Fig. 3. When there were no metal partitions between the TX and RXs, we had a line-of-sight (LOS) environment, as seen in Fig. 4(a). When there were partitions, we had a non-LOS (NLOS) one, as shown in Fig. 4(b).

Two stepping motors were used on the RX side to move the two RX arrays along the $x$ - or $y$-axis during the experiments. These motors were controlled by a personal computer. Each step of the motors corresponded to $0.0088 \mathrm{~cm}$, and the RX arrays were stopped at every 10 steps (equal to $0.088 \mathrm{~cm})$. The channels were measured at intervals of $0.088 \mathrm{~cm}$, and we had a total of 500 spatial measuring points. As a result, channel response matrices were obtained for each case of direction of RX motion and LOS/NLOS condition. A large number of channel data was measured to find reliable BER. Note that the measurement campaign was conducted while no one was in the room to ensure statistical stationarity of propagation. 


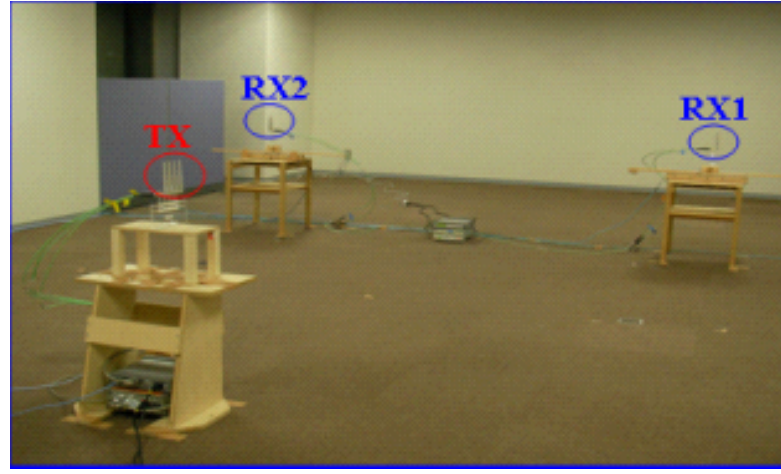

(a) LOS environment.

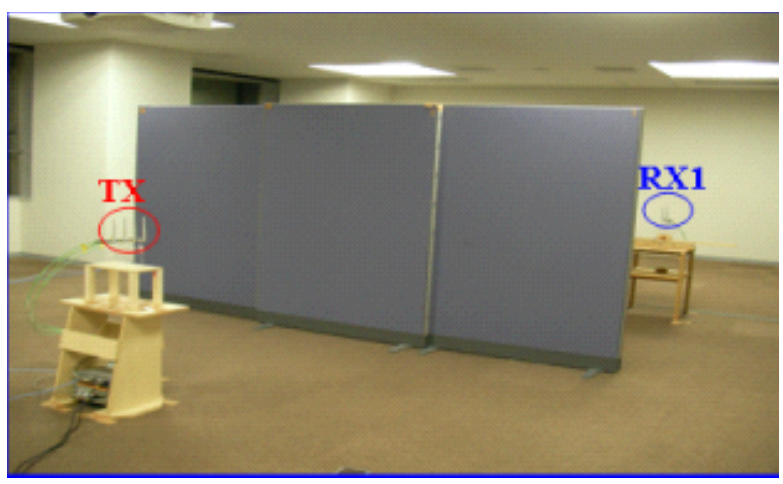

(b) NLOS environment

Fig. 4 Measurement environments. (Reprinted from Bui et al. [26] (C) 2013 IEEE)

\section{Channel Autocorrelations and Doppler Spectra}

We assumed that RXs were moving at constant velocity $v$. We will discuss our examination of channel autocorrelations and Doppler spectra in this section using the measured data. As stated in the previous section, the channels were measured at intervals of $0.088 \mathrm{~cm}$. Assuming that the time interval between adjacent measuring points is $0.833 \mathrm{~ms}$, then the velocity is $v=0.088 \mathrm{~cm} / 0.833 \mathrm{~ms}=1.06 \mathrm{~m} / \mathrm{s}$. This motion causes maximum Doppler frequency $f_{\mathrm{D}}$ of $18.6 \mathrm{~Hz}$ for the center frequency of $5.275 \mathrm{GHz}$.

We have considered two cases in this paper:

(a) The NLOS environment and RX motion along the $x$ axis

(b) The LOS environment and RX motion along the $y$-axis

The channel autocorrelations and Doppler spectra for RX1 are shown in Fig. 5. We had $4 \times 2$ MIMO channels. The channel autocorrelations were obtained by averaging over the eight channels as well as the spatial domain and 1,601 frequency domain data. Autocorrelation assuming the Jakes' model is also provided in Fig. 5. If we divide the measuring distance (abscissa) by velocity $v=1.06 \mathrm{~m} / \mathrm{s}$, we have the channel autocorrelations versus time. The Doppler spectra of both the measured data and the Jakes' model were cal- culated by applying a 450-point discrete Fourier transform (DFT) process to the time domain channel autocorrelations after multiplying them by the Hamming window [34].

The Doppler spectrum in the NLOS environment shown in Fig. 5(a) has peaks at $\pm 18.6 \mathrm{~Hz}$, which correspond to $\pm f_{\mathrm{D}}$. This is similar to the Doppler spectrum of the Jakes' model. However, we can see another peak at about $7.5 \mathrm{~Hz}$. A strong reflection corresponding to the peak was considered to be at the site, which is different from the Jakes' model.

Both autocorrelation and the Doppler spectrum were completely different from those of the Jakes' model for LOS and RX motion along the $y$-axis (Fig. 5(b)). We can see that the power spectral density is mainly distributed around the Doppler frequency of $7 \mathrm{~Hz}$, which is caused by a direct wave from the TX in the LOS environment. If we compensate for the Doppler frequency of $7 \mathrm{~Hz}$, the effect of time-varying channels is largely mitigated. This will be shown in the next section.

\section{Accuracy of Channel Prediction}

We investigated the BER performance of multi-user MIMO downlink transmission using the measured channel data. We conducted simulations of the multi-user MIMO E-SDM system by assuming the parameters listed in Table 1 . Some parameters and transmission schemes stated below are the same as those in Bui et al. [26]. Because the TX had four antennas and each RX had two antennas, we had either single-stream or two-stream transmission for each RX. We assumed that the data rate for each MS was fixed constantly at $4 \mathrm{bps} / \mathrm{Hz}$ (4 bits/symbol). The modulation scheme was either 16QAM for the single-stream transmission or QPSK for the two-stream one. The control of resources for ESDM transmission, viz., determining the number of streams, modulation scheme, and transmit power, was done in such a way that the Chernoff upper bound of BER for each MS had the lowest value [8]. The total transmit power per MS was equal. The uplink channels in this study were assumed to be perfectly estimated at the TX using ACK packets, and the effective downlink channels for E-SDM transmission were also assumed to be perfectly estimated at both RXs. Each RX determined the MMSE weights using its own effective downlink channels.

Figure 6 shows the average BER performance of RX1 versus normalized TX power for RX motion along the $x$ axis in the NLOS case. The normalized TX power is the TX power per MS normalized to the power yielding average $E_{s} / N_{0}$ of $0 \mathrm{~dB}$ in single-user SISO-LOS measurements in an anechoic chamber. Here, $E_{s}$ is the received signal energy per symbol, and $N_{0}$ is the noise power density. In addition to the BER performance with the prediction of channels for different values of AR-model order $p$, performance without prediction and that of the ideal case are also plotted in the figure. The ideal case in the figure indicates behavior in accurately predicting channels. As indicated in Table 1, all the curves are for delay $\tau$ of $5 \mathrm{~ms}$ from the ACK packet. The 

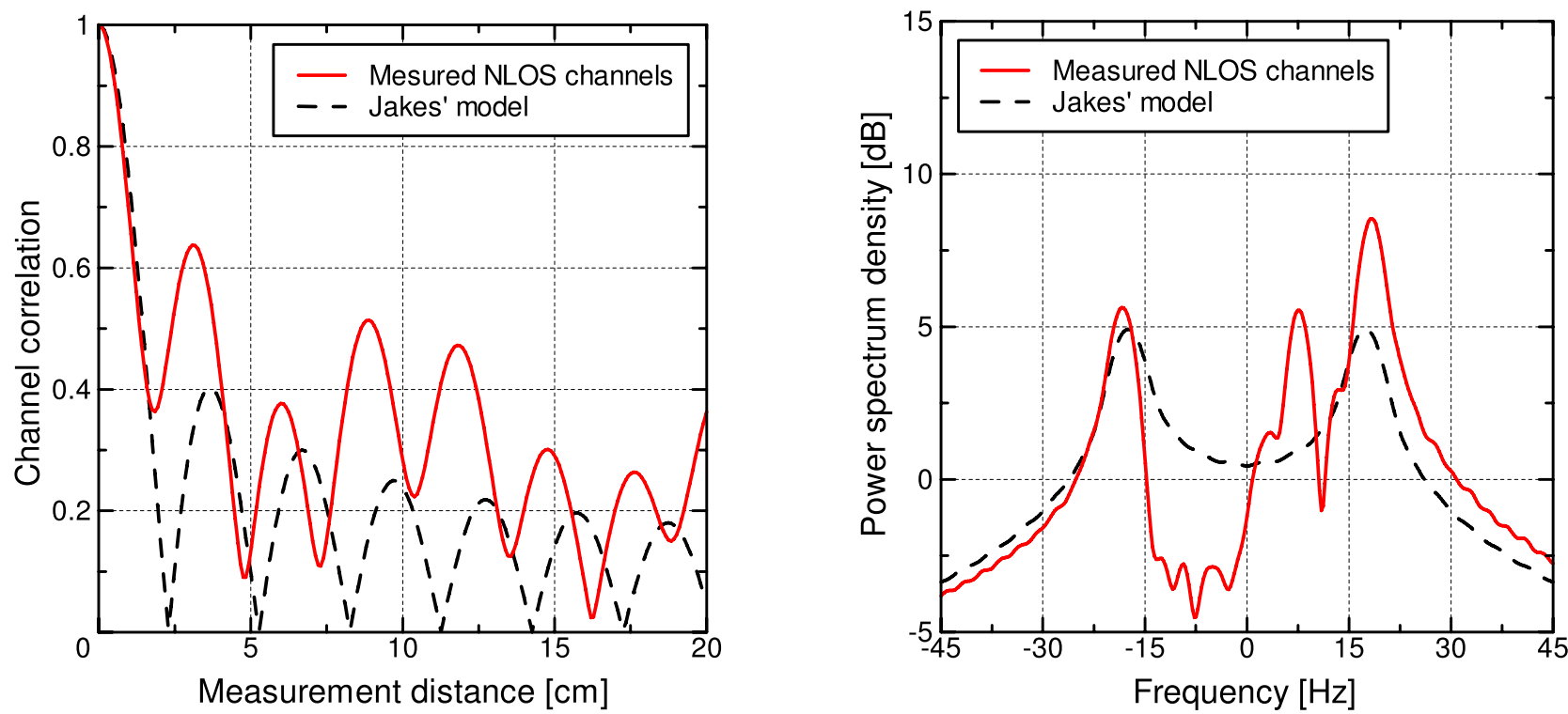

(a) NLOS environment with RX motion along $x$-axis.
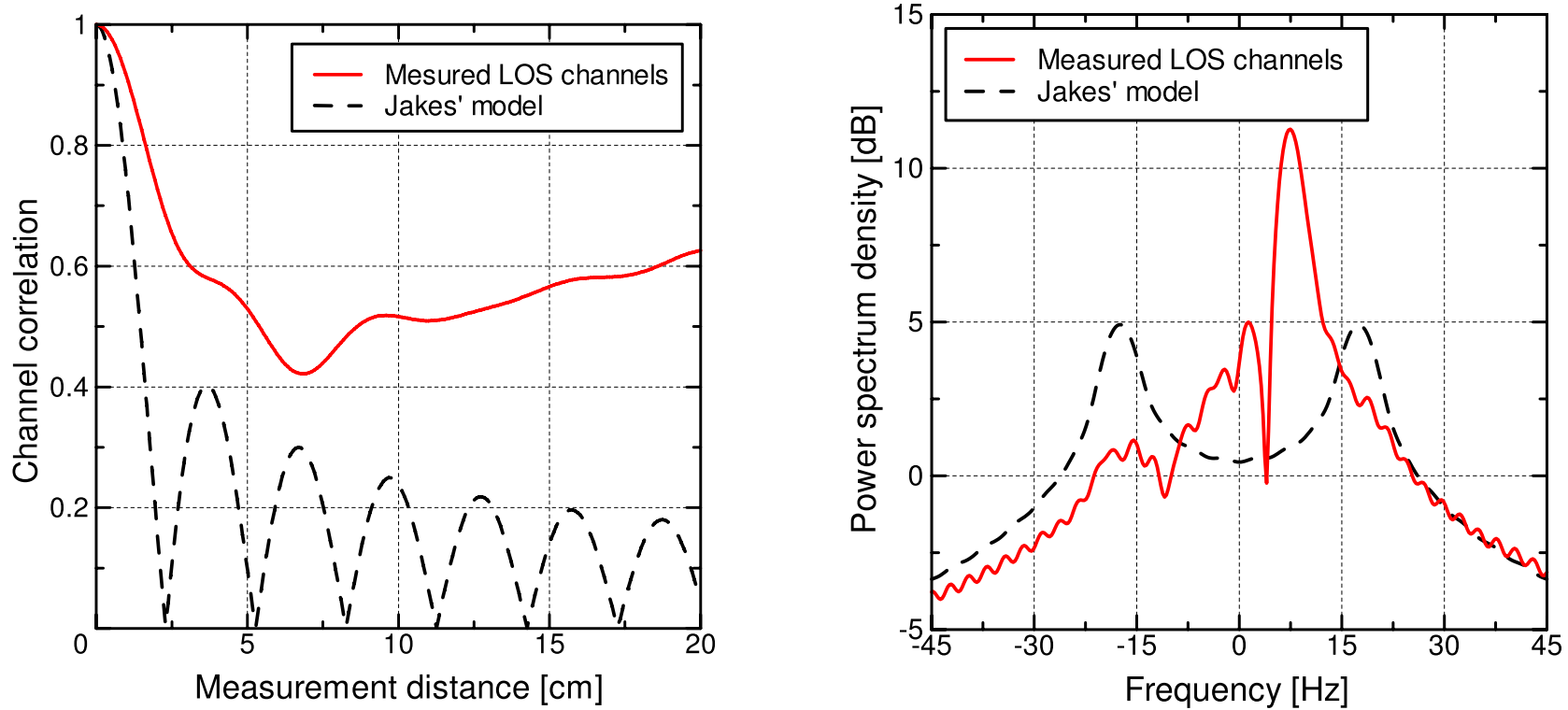

(b) LOS environment with $\mathrm{RX}$ motion along $y$-axis.

Fig. 5 Channel autocorrelations and Doppler spectra for RX1.

figure reveals that when we do not use the scheme of channel prediction, we have a high error floor. This means that if we use outdated channels when ACK packets are received, we have poor BER performance. The distance traveled during $5 \mathrm{~ms}$ for the velocity of $1.06 \mathrm{~m} / \mathrm{s}$ or $f_{\mathrm{D}}$ of $18.6 \mathrm{~Hz}$ is $0.53 \mathrm{~cm}$ or 0.093 wavelengths. It should be noted that only a fraction of channel transition significantly affected the BER performance even though the RX weights were determined by the MMSE criterion using CSI without delay. As can be seen from Fig. 5(a), we have strong components at the Doppler frequencies of \pm 18.6 and $7.5 \mathrm{~Hz}$. These cause channel changes and deteriorate the BER performance. When we use channel prediction, on the other hand, BER is re- duced depending on AR-model order $p$. Especially, when $p=3$, BER is reduced close to the ideal case. It should be noted that the channel autocorrelations required for the ARmodel-based prediction of channels are obtained assuming the Jakes' model. As can be seen from the Doppler spectra in Fig. 5(a), the actual radio environment is different from that of the Jakes' model. However, prediction is very effective for $p=3$. We assumed frame duration $T_{f}$ of $10 \mathrm{~ms}$ in the simulations, which corresponds to $1.06 \mathrm{~cm}$ for the velocity of $1.06 \mathrm{~m} / \mathrm{s}$. We used autocorrelations up to $2.12 \mathrm{~cm}$ apart when $p=3$. We can see from Fig. 5(a) that the autocorrelations in the Jakes' model and the actual channels are the same up to about $2 \mathrm{~cm}$. We conjectured that this was 
Table 1 Simulation parameters.

\begin{tabular}{c|c}
\hline No. of MSs & 2 \\
\hline No. TX \& RX antennas & $4 \times 2$ \\
\hline Data rate for each MS & 4 bits/symbol \\
\hline Modulation schemes & QPSK, 16QAM \\
\hline Resource control & $\begin{array}{c}\text { Minimum BER criterion based } \\
\text { on Chernoff upper bound }\end{array}$ \\
\hline Data burst length & 128 symbols \\
\hline Franme duration $\left(T_{f}\right)$ & $10 \mathrm{~ms}$ \\
\hline Delay from ACK $(\tau)$ & $5 \mathrm{~ms}$ \\
\hline Max. Doppler frequency $\left(f_{\mathrm{D}}\right)$ & $18.6 \mathrm{~Hz}$ \\
\hline Channels & $\begin{array}{c}\text { Frequency flat-fading estimated } \\
\text { perfectly at TX, RX1 and RX2 }\end{array}$ \\
\hline Thermal noise & Additive white Gaussian noise \\
\hline RX signal processing & MMSE weight
\end{tabular}

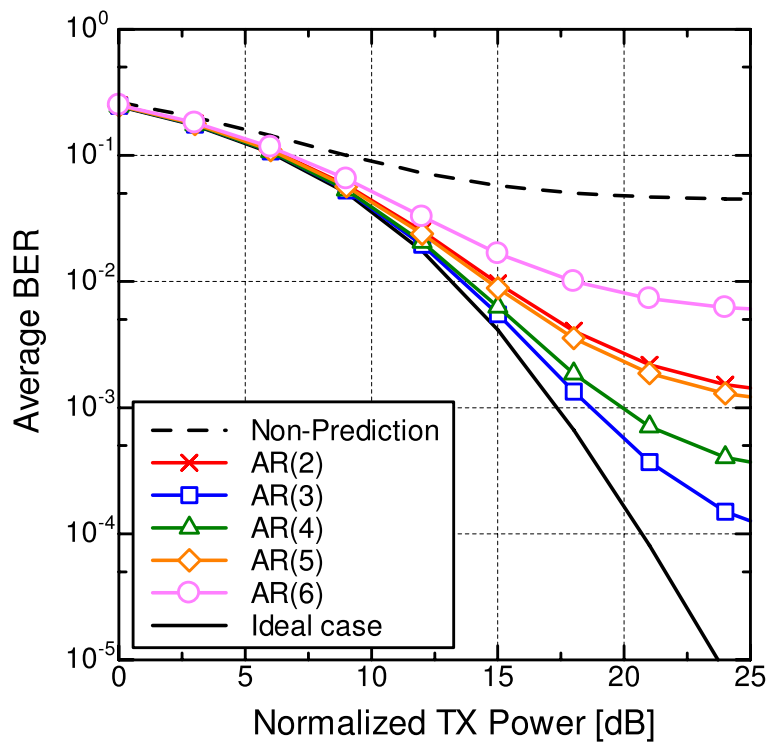

Fig. 6 BER performance with channel prediction assuming the Jakes' model (1). NLOS, RX1, RX motion along $x$-axis, $v=1.06 \mathrm{~m} / \mathrm{s}, f_{\mathrm{D}}=$ $18.6 \mathrm{~Hz}$.

the reason why we could reduce BER assuming the Jakes' model.

Figure 7 shows the average BER performance of RX1 versus normalized TX power for RX motion along the $y$-axis in the LOS environment. We can see that BER is reduced more than that in Fig. 6. As stated in the previous section, the Doppler spectrum is almost concentrated around $7 \mathrm{~Hz}$ in this scenario, and the effect of the time-varying channels can easily be mitigated. Also in this LOS environment, the AR-model-based prediction of channels with $p=3$ reduces BER so that it is close to that of the ideal case. It is interesting that although channel autocorrelation for the LOS case is different from that for the Jakes' model, as seen from Fig. 5(b), the BER performance is improved assuming the Jakes' model.

The optimum value of $p$ was 3 for both the cases. The value, however, may be different depending on environments and parameters such as $T_{f}$ and $\tau$. The method of determining the optimum $p$ has not been available.

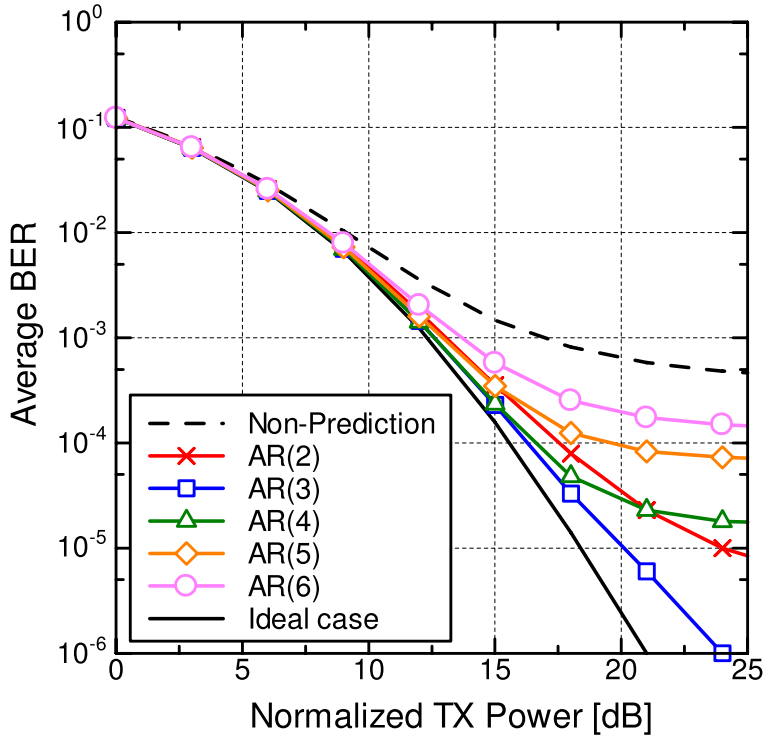

Fig. 7 BER performance with channel prediction assuming the Jakes' model (2). LOS, RX1, RX motion along $y$-axis, $v=1.06 \mathrm{~m} / \mathrm{s}, f_{\mathrm{D}}=$ $18.6 \mathrm{~Hz}$.

\section{Conclusions}

We investigated the behavior of multi-user MIMO systems in time-varying environments. We found that BER was seriously degraded depending on channel scenarios. However, if we predicted future channels using the AR-model-based scheme, performance was largely improved. We also found that we could assume the Jakes' model for calculations of AR coefficients. The reason for this was not clear, and this is an issue that needs to be resolved in future work. Here, we treated the prediction range of $0.53 \mathrm{~cm}$ or 0.093 wavelengths, and evaluated the average BER. The prediction of channels for longer ranges also remains for future work.

\section{References}

[1] E. Biglieri, R. Calderbank, A. Constantinides, A. Goldsmith, A. Paulraj, and H. V. Poor, MIMO Wireless Communications, Cambridge University Press, Cambridge, 2007.

[2] A.J. Paulraj, D.A. Gore, R.U. Nabar, and H. Bölcskei, "An overview of MIMO communications - A Key to gigabit wireless," Proc. IEEE, vol.92, no.2, pp.198-218, Feb. 2004.

[3] "Special issue on multiple-input multiple-output (MIMO) technology,” IEEE Trans. Antennas Propag., vol.60, no.2, Feb. 2012.

[4] "Special issue on adaptive antennas," IEEE Trans. Antennas Propag., vol.AP-24, no.5, Sept. 1976.

[5] "Special issue on adaptive signal processing technology in antennas," IEICE Trans. Commun., vol.E78-B, no.11, Nov. 1995.

[6] R.A. Monzingo, R.L. Haupt, and T.W. Miller, Introduction to Adaptive Arrays, 2nd ed., SciTech Publishing, Raleigh, 2011.

[7] E. Telatar, "Capacity of multi-antenna Gaussian channels," Eur. Trans. Telecomm., vol.10, no.6, pp.585-589, Nov./Dec. 1999.

[8] K. Miyashita, T. Nishimura, T. Ohgane, Y. Ogawa, Y. Takatori, and K. Cho, "High data-rate transmission with eigenbeam-space division multiplexing (E-SDM) in a MIMO channel," Proc. IEEE VTC 2002Fall, vol.3, pp.1302-1306, Sep. 2002.

[9] G. Lebrun, J. Gao, and M. Faulkner, "MIMO transmission over a 
time-varying channel using SVD," IEEE Trans. Wireless Commun. vol.4, no.2, pp.757-764, March 2005.

[10] S.H. Ting, K. Sakaguchi, and K. Araki, "A robust and low complexity adaptive algorithm for MIMO eigenmode transmission system with experimental validation," IEEE Trans. Wireless Commun., vol.5, no.7, pp.1775-1784, July 2006.

[11] D. Gesbert, M. Kountouris, R.W. Heath Jr., C.B. Chae, and T. Sälzer, "From single user to multiuser communications: Shifting the MIMO paradigm," IEEE Signal Process. Mag., vol.24, no.5, pp.36-46, Sept. 2007

[12] R. Kudo, Y. Takatori, A. Ohta, K. Nishimori, and S. Kubota, "Evaluation of downlink transmission performance for time varying channel in actual indoor environment in single and multi user MIMO systems," IEICE Technical Report, AP2006-140, Feb. 2007.

[13] K. Saito, Y. Inoue, T. Kawamura, and H. Andoh, "Field experiments on 1-Gbps data transmission using 4-by-2 multi-user MIMO with cross-polarized linear antenna array in LTE-Advanced downlink," Proc. IEEE VTC 2013-Spring, June 2013.

[14] K. Huang, R.W. Heath, Jr., and J.G. Andrews, "Limited feedback beamforming over temporally-correlated channels," IEEE Trans. Signal Process., vol.57, no.5, pp.1959-1975, May 2009.

[15] J.W. Wallance and M.A. Jensen, "Time-varing MIMO channels: Measurement, analysis, and modeling," IEEE Trans. Antennas Propag., vol.54, no.11, pp.3265-3273, Nov. 2006.

[16] A.L. Anderson, J.R. Zeidler, and M.A. Jensen, "Stable transmission in the time-varying MIMO broadcast channel," EURASIP J. Adv. Signal Process., vol.2008, no.Article ID 617020, 2008.

[17] A.L. Anderson, J.R. Zeidler, and M.A. Jensen, "Reduced-feedback linear precoding with stable performance for the time-varying MIMO broadcast channel," IEEE J. Sel. Areas Commun., vol.26, no.8, pp.1483-1493, Oct. 2008.

[18] A. Duel-Hallen, "Fading channel prediction for mobile radio adaptive transmission systems," Proc. IEEE, vol.95, pp.2299-2313, Dec. 2007.

[19] C. Komninakis, C. Fragouli, A.H. Sayed, and R.D. Wesel, "Multiinput multi-output fading channel tracking and equalization using Kalman estimation," IEEE Trans. Signal Process., vol.50, no.5, pp.1065-1076, May 2002.

[20] A.S. Khrwat, B.S. Sharif, C.C. Tsimenidis, and S. Boussakta, "Channel prediction for precoded spatial multiplexing multipleinput multiple-output systems in time-varying fading channels," IET Signal Process., vol.3, no.6, pp.459-466, Nov. 2009.

[21] H. Hijazi and L. Ros, "Joint data QR-detection and Kalman estimation for OFDM time-varying Rayleigh channel complex gains," IEEE Tran. Commun., vol.58, no.1, pp.170-178, Jan. 2010.

[22] H.P. Bui, H. Nishimoto, T. Nishimura, T. Ohgane, and Y. Ogawa, "Performance of MIMO E-SDM systems using channel prediction in actual time-varying indoor fading environments," IEICE Trans. Commun., vol.E91-B, no.6, pp.1713-1723, June 2008.

[23] M. Chen and M. Viberg, "Long-range channel prediction based on nonstationary parametric modeling," IEEE Trans. Signal Process., vol.57, no.2, pp.622-634, Feb. 2009.

[24] J.P. Leite, P. Henrique, P. Carvalho, and R.D. Vieira, "Channel prediction on the downlink of precoded multiuser MIMO OFDM systems using the set-membership affine projection filtering," Proc. IEEE VTC2011-Fall, Sept. 2011.

[25] J. Chang, I.T. Lu, and Y.X. Li, "Adaptive codebook-based channel prediction and interpolation for multiuser multiple-input multipleoutput-orthogonal frequency division multiplexing systems," IET Commun., vol.6, no.3, pp.281-288, Feb. 2012.

[26] H.P. Bui, Y. Ogawa, T. Nishimura, and T. Ohgane, "Performance evaluation of a multi-user MIMO system with prediction of timevarying indoor channels," IEEE Trans. Antennas Propag., vol.61, no.1, pp.371-379, Jan. 2013.

[27] S.O. Haykin, Adaptive Filter Theory, Third Edition, Prentice Hall, 1996.

[28] A. Doufexi, S. Armour, M. Butler, A. Nix, D. Bull, J. McGeehan, and P. Karlsson, "A comparison of the HIPERLAN/2 and IEEE 802.11a wireless LAN standards," IEEE Commun. Mag., vol.40, no.5, pp.172-180, May 2002.

[29] D. Astály, E. Dahlman, A. Furuskär, Y. Jading, M. Lindström, and S. Parkvall, "LTE: The evolution of mobile broadband," IEEE Commun. Mag., vol.47, no.4, pp.44-51, April 2009.

[30] K. Etemad, "Overview of mobile WiMAX technology and evolution," IEEE Commun. Mag., vol.46, no.10, pp.31-40, Oct. 2008.

[31] L.U. Choi and R.D. Murch, "A transmit preprocessing technique for multiuser MIMO systems using a decomposition approach," IEEE Trans. Wireless Commun., vol.3, no.1, pp.20-24, Jan. 2004

[32] Q.H. Spencer, A.L. Swindlehurst, and M. Haardt, "Zero-forcing methods for downlink spatial multiplexing in multiuser MIMO channels," IEEE Trans. Signal Process., vol.52, no.2, pp.461-471, Feb. 2004.

[33] Q.H. Spencer, C.B. Peel, A.L. Swindlehurst, and M. Haardt, "An introduction to the multi-user MIMO downlink," IEEE Commun. Mag., pp.60-67, Oct. 2004.

[34] H.P. Bui, H. Nishimoto, Y. Ogawa, T. Nishimura, and T. Ohgane, "Channel characteristics and performance of MIMO E-SDM systems in an indoor time-varying fading environment," EURASIP J Wireless Commun. Network., vol.2010, Article ID 736962, 2010.

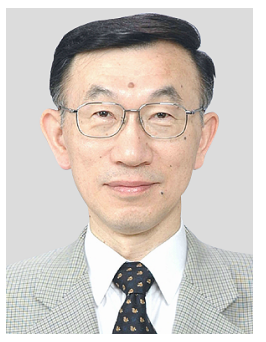

Yasutaka Ogawa received his B.E., M.E. and Ph.D. from Hokkaido University, Sapporo, Japan, in 1973, 1975, and 1978. Since 1979, he has been with Hokkaido University, where he is currently a full Professor at the Graduate School of Information Science and Technology. From 1992-1993, he was with the ElectroScience Laboratory of Ohio State University, U.S.A., as a Visiting Scholar, on leave from Hokkaido University. His interests are in adaptive antennas, mobile communications, super-resolution techniques, and MIMO systems. Dr. Ogawa received the Yasujiro Niwa outstanding paper award in 1978, the Young Researchers' Award of IEICE Japan in 1982, the Best Paper Award from IEICE Japan in 2007, and the Best Magazine Paper Award in 2011 from the IEICE Communications Society. Dr. Ogawa is a Fellow of the IEEE.

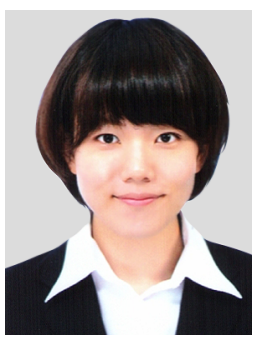

Kanako Yamaguchi received her B.E from Hokkaido University in Sapporo, Japan, in 2012. She is currently a master course student at the Graduate School of Information Science and Technology, Hokkaido University. Her interest are in MIMO systems and mobile communications. 


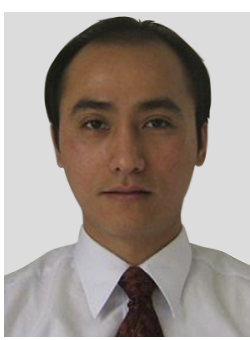

Huu Phu Bui received his B.S. in electronics engineering from Danang University in 1997 and his M.S. in electronics engineering from Hochiminh City University in 2002, both in Vietnam. He received his Ph.D. in information science and technology from Hokkaido University, Japan in 2007. From 1997 to 2007, he was with the Radio Frequency Directorate of the Ministry of Information and Communications, Vietnam. From 2007 to 2011, he was with Hochiminh City University of Science, Vietnam. He is currently a Vice Director of the Vietnam National Key Laboratory of Digital Control and System Engineering at the Hochiminh City University of Technology. From 2007 to 2009, he was a postdoctoral researcher at Hokkaido University, Japan. His research interests are in channel prediction and signal processing for MIMO systems. He received the IEEE VTS Japan Chapter Young Researcher's Encouragement Award in 2006.

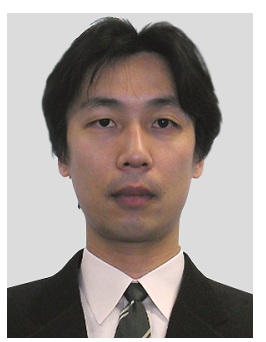

Toshihiko Nishimura received his B.S in physics in 1992, his M.S. in physics in 1994, and his Ph.D. in electronics engineering in 1997 all from Hokkaido University, Sapporo, Japan. He joined the Graduate School of Engineering (presently reorganized as the Graduate School of Information Science and Technology) at Hokkaido University in 1998, where he is currently an Assistant Professor of the Graduate School of Information Science and Technology. His current research interests are in MIMO systems using smart antenna techniques. Dr. Nishimura received the Young Researchers' Award of IEICE Japan in 2000, the Best Paper Award from IEICE Japan in 2007, and the Best Magazine Paper Award in 2011 from the IEICE Communications Society. Dr. Nishimura is a member of the IEEE.

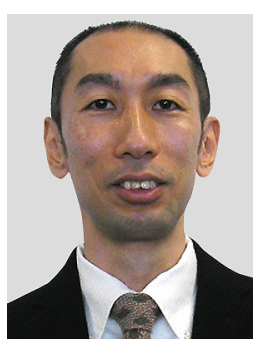

Takeo Ohgane received his B.E., M.E., and $\mathrm{Ph} . \mathrm{D}$. in electronics engineering from Hokkaido University, Sapporo, Japan, in 1984, 1986, and 1994. He was with the Communications Research Laboratory of the Ministry of Posts and Telecommunications from 1986 to 1992 . He was on assignment at the ATR Optical and Radio Communications Research Laboratory from 1992 to 1995 . Since 1995 , he has been with Hokkaido University, where he is an Associate Professor. He was at the Centre for Communications Research of the University of Bristol, U.K., as a Visiting Fellow from 2005-2006. His interests are in MIMO signal processing for wireless communications. Dr. Ohgane received the Young Researchers' Award of IEICE Japan in 1990, the IEEE AP-S Tokyo Chapter Young Engineer Award in 1993, the Best Paper Award from IEICE Japan in 2007, and the Best Magazine Paper Award in 2011 from the IEICE Communications Society. Dr. Ohgane is a member of the IEEE. 